\title{
Review on Hands Gestures Using American Sign Languages
}

\author{
Krutika S. Kale*1, Prof. Milind B. Waghmare ${ }^{2}$
}

${ }^{*}$ Department of Computer Science and Engineering, Government College of Engineering Amravati, India ${ }^{2}$ Professor, Department of Computer Science and Engineering, Government College of Engineering Amravati, India

\section{ABSTRACT}

\section{Article Info}

Volume 7, Issue 3

Page Number: 228-232

Publication Issue :

May-June-2021

\section{Article History}

Accepted : 15 May 2021

Published : 22 May 2021
The Disability of speech impairment affects the ability to speech and communicates with others, and such disability makes person to use other medium to communicate such as sign language. And it is a challenge to make communication between people who understand sign language and person who doesn't understand sign language. Sign language is no yet so popular method among the hearing people. To overcome this sign language issue, a sign language detection technology is used by using image classification and machine learning. Sign language recognition points to covert information in the form of sign language to user who have a little knowledge regarding sign language in the form of text or voice and this will be a huge support for communication between deaf-mute and normal people.

Keywords : Sign Language Recognition, Human Computer Interaction, image processing, computer science, Hand gesture recognition, machine learning.

\section{INTRODUCTION}

Hand gesture recognition using visionbased technologies plays an integral role in humancomputer interaction (HCI) The keyboard and mouse are the two most crucial functions of humancomputer interaction in recent decades. However, new forms of HCI methods have been required because of the exponential increase of hardware /software. In the field of HCI, innovations like speech recognition and gesture recognition attract a lot of attention Gestures can be used to communicate with humans and computers. Gesture detection differs from conventional hardware-based approaches in that it allows humans to communicate with computers. The user intent is determined by gesture recognition, which recognizes the gesture or action of the body or body parts. Many researchers have worked for decades to develop hand motion recognition technologies. Hand motion recognition is useful in a variety of applications, including artificial reality (virtual reality), handicapped sign language interpreters, and robot control Gestures, like speech, are a natural form of human communication. They might be the most natural form of expression. This is the fact that infants use hand gestures to communicate emotions and desire to speak.

Human movements can be thought of as a pattern recognition challenge. The desired message can be reconstructed if a programmer can track and discern certain human motion patterns. The detection of staticsign movements for representing digits or letters is effective. 
For the vast majority of the population, oral communication is the primary means of contact. A substantial large number of people would be unable to speak without the use of oral communication. Despite the fact that oral communication exists, a segment of the individual is not able to communicate with the rest of the people. People who are mute are not able to talk with the help of verbal communication and, therefore, SL comes to support these types of people. Sign language uses gestures of hand, static-hand signals and facial expressions to interact in the same way as spoken language does. There are several different varieties of sign languages, just like there are many different types of spoken languages.

keyboard and mouse have played a major role in human-computer interaction in recent decades. However, new forms of HCI methods have been required as a result of the rapid growth of hardware and software. In the field of HCI, innovations like speech recognition and gesture recognition attract a lot of attention Gestures can be used to communicate with humans and computers. Gesture detection differs from conventional hardware-based approaches in that it allows humans to communicate with computers. The user intent is determined by gesture recognition, which recognizes the gesture or action of the body or body parts. Many researchers have worked for decades to develop hand motion recognition technologies. Hand motion recognition is useful in a variety of applications, including artificial reality (virtual reality), handicapped sign language interpreters, and robot control Gestures, like speech, are a natural form of human communication. They might be the most natural form of expression. This is the fact that infants use hand gestures to communicate emotions and desire to speak.

Human movements can be thought of as a pattern recognition challenge. The desired message can be recreated if a programmer can track and discern certain human motion patterns. The detection of staticsign movements used to represent alphabets and numbers has been effective. This scheme, however, can be expanded to recognize words and sentences as well. We've used American Sign Language (ASL) as the sign language for which we're looking for gestures.

For the vast majority of the population, spoken language is the primary means of contact. A substantial majority of the population would be unable to speak without the use of spoken language. Despite the fact that spoken language exists, a segment of the population is unable to communicate with the rest of the population. People who are mute are not able to communicate using speaking languages and therefore sign language comes to support this type of people.

Sign language uses facial expressions, static hand signals, and hand gestures to interact in the same way as spoken language does. There are several different varieties of sign languages, just like there are many different types of spoken languages.

\section{RELATED WORK}

Camera, hand belts and Data gloves are the most common methods of obtaining feedback from a consumer who has been detected [1]. [2] They process data using a sliding window technique, which allows them to compute on data while it is generated or obtained. This gesture recognition method makes use of data gloves to collect input. [3] This software uses hand motion recognition to read the accelerometer and gyroscope in a smartphone and send a control order to the wireless remote-control device.[4] To examine palm gestures, bluetooth, a hand belt with a gyroscope and accelerometer was used. In [5,] they used a Senz3D camera to gather as much detail and colour as possible. [6] They used a Bumblebee2 stereo camera and implemented an actual optimization method for incoming hand pictures and this application detects hands and 
perceives the foreground object. [7] employed a monocular digital lens. [8] An integrated digital mouse device based on hand gesture recognition is proposed in this article. Easy web cameras were used to integrate systems in cost-effective models like [9]. [10] It is based on PCA to interpret sign language in Vietnamese Sign Language against a standardized context which compares its efficacy of Euclidean and Mahalanobis distance classifiers.

[11] It is proposed to grow a actual Kinect-based structured HGR framework that includes gesture classification, hands detection and data processing. In this proposed scheme, movement direction is derived as just a special function, and the recognition algorithm is Support Vector Machine (SVM). The hand sizes, textures, and depths are portrayed as superpixels, which essentially preserve the general shapes and colours of the movements to be remembered. A modern data value, superpixel earth mover's distance, has been utilized to compute the dissimilarity in between arm movements based on this representation [12]. In comparison to standard images, depth cameras offer extra rich data to every pixels of screen resolution [13], [14]. This may not completely address the underlying issue. [15] used a light and dark layout for virtual world symbols to address the context problem (monochrome glove). Though built-in webcams do not have depth knowledge, they do save money on computation. [16] Is study of total forty six articles on gesture recognition, both static and dynamic gesture recognition in basic and complex environments. Skin colour for attribute extraction is often used in works that do not need specific hardware, but identifying the gesture and removing background noise can be difficult when the background has dynamic lighting.

[17] address a number of visionbased SL Recognition Systems that researchers used to compare various approaches. The downside to using a web camera, or merely a camera, is that it necessitates a thorough preprocessing procedure in order to achieve the function. Hidden Markov Models (HMM) are the oldest and most widely used classification tool, and have been used for years in the development of Sign Language Recognition. [18] address common strategies for SLR device pre-processing, segmentation, feature extraction, and classification separately. When using a skin colour threshold algorithm to segment the hand field, HSV is a colour space that is normally resistant to lighting conditions. [19] demonstrate a pca-Based feature selection method which is a common dimension reduction technique. Its aim is to keep several valuable characteristics such as high dimensional data while eliminating distortion and irrelevant attribute in order to improve data processing speed. [20] For sign language, an actual visionbased stable hand gesture recognition program was created. A Universal Serial Bus device attached to a desktop was used to capture all of the data, and no additional objects (such as gloves) were needed. The suggested scheme uses a skin colour method in Hue, Saturation, Value colour to locate the hand gesture's ROI. Using Principal Component Analysis, eight images are segmented from every model after all pre-processing work was completed. Support Vector Machine was used as the machine learning method for identification.[21] Created a vision-based program that converts sign language to documents, allowing people who know sign language and people who don't know sign language to communicate with one another. This works by extracting spatial - temporal attributes via video sequences. CNN is then used to identify visual characteristics. Then, for practicing on different amplitude characteristics, they use an RNN (Recurrent Neural Network). The American Sign Language Dataset was used in this study.

\section{APPROACH REGARDING PROPOSED METHODOLOGY}

The two forms of sign language recognition are glove-based recognition and vision-based recognition. 
A CNN is a strong neural network that uses filters to extract features from images. Furthermore, it extracts attributes from images in such a manner that pixel location detail is preserved. A convolution is a mathematical operation that is performed on a matrix. This matrix is usually the representation in the form of pixels or numbers. Convolution operation is used to remove features from images; it captures spatial features better than artificial neural network.

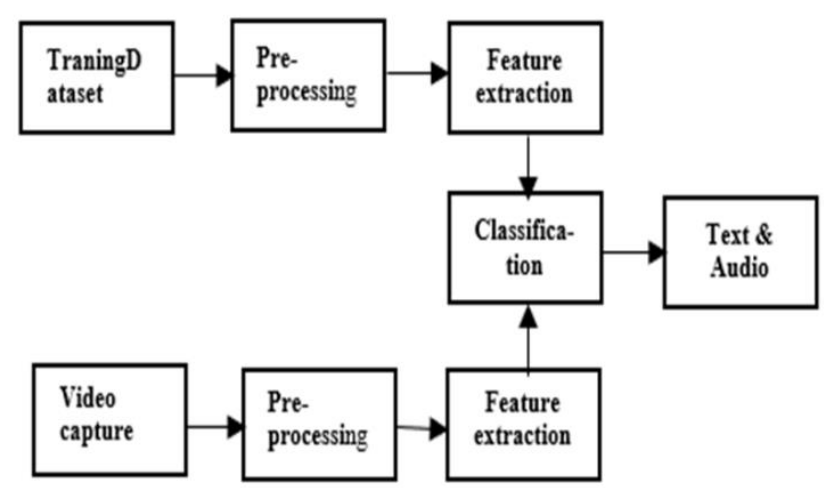

Figure 1. Flowchart of Proposed Methodology

\section{IV.CONCLUSION}

Image recognition and machine learning are combined to create a sign language detection system. Sign language recognition helps to convert sign language into text or voice for people who only have a basic understanding of it, which would be a huge support to deaf-mute and hearing people in communicating. The sign language and gesture recognition system is a module that allows deaf and dumb people to communicate in a simple and satisfactory manner. The module allows for two-way conversation, allowing common people and disabled people to communicate more easily. This method is a great way to help people with speech and vocal disorders communicate more easily. By using an image processing algorithm, we must provide an application to society that will facilitate contact between deaf and mute people. It can be launched as an application in any minimal environment and therefore has a near-zero cost since it uses an imagebased approach.

\section{REFERENCES}

[1]. Granit Luzhnica, Elizabeth Lex, Viktoria Pammer, "A Sliding Window Approach to Natural Hand Gesture Recognition using a Custom Data Glove," in 3D User Interfaces (3DUI), 2016 IEEE Symposium, New York : IEEE 2016, pp. 81-90, 2016 Mar 19.

[2]. Ji-Hwan Kim, Nguyen Duc Thang, Tae-Seong Kim, "3-D Hand Motion Tracking and Gesture Recognition Using a Data Glove," in Industrial Electronics, 2009 IEE International Symposium, New York : IEEE 2009, pp. 5-6, 2009 July 5

[3]. Hung CH, Bai YW, Wu HY, " Home outlet and LED array lamp controlled by a smartphone with a hand gesture recognition," in Consumer Electronics (ICCE), 2016 IEEE International Conference, New York : IEEE 2016, pp. 5-6, 2016 Jan 7.

[4]. Hung CH, Bai YW, Wu HY, "Home appliance control by a hand gesture recognition belt in LED array lamp case," in Consumer Electronics (GCCE), 2015 IEEE 4th Global Conference, New York : IEEE 2015, pp. 599-600, 2015 Oct 27.

[5]. She Y, Wang Q Jia Y, Gu T, He Q Yang B, “A real-time hand gesture recognition approach based on motion features of feature points," in Computational Science and Engineering (CSE), 2014 IEEE 17th International Conference, New York : IEEE 2014, pp. 1096-1102, 2014 Dec 19.

[6]. Lee DH, Hong KS, "A Hand gesture recognition system based on difference image entropy," in Advanced Information Management and service (IMS), 2010 6th International Conference, New York : IEEE 2010, pp. 410-413, 2010 Nov 30.

[7]. Dulayatrakul J, Prasertsakul P, Kondo T, Nilkhamhang I, "Robust implementation of hand gesture recognition for remote human-machine interaction," in Information Technology and Electrical Engineering (ICITEE), 2015 7th 
International Conference, pp. 247-252, 2015 Oct 29.

[8]. Tsai TH, Huang CC, Zhang KL, "Embeded virtual mouse system by using hand gesture recognition," in Consumer Electronics-Taiwan (ICCE-TW), 2015 IEEE International Conference, Taiwan Taipei, New York : IEEE 2015, pp. 352-353, 2015 Jun 6.

[9]. Hussain I, Talukdar AK, Sarma KK, "Hand gesture recognition system with real-time palm tracking, " in India Conference (INDICON), 2014 Annual IEEE New York : IEEE 2014, pp.1-6, 2014 Dec 11.

[10]. Huong TN, Huu TV, Le Xuan T, " Static hand gesture for Vietnamese sign languages (VSL) using principle components analysis," in communications, Management and Telecommunication (ComMan Tel), 2015 International Conference, pp.138-141, 2015 Dec 28.

[11]. Chen Y, Luo B, Chen YL, Liang G, Wu X, "A realtime dynamic hand gesture recognition system using Kinect sensor, " in Robotics and Biomimetics (ROBIO), 2015 IEEE International Conference, New York : IEEE 2015, pp.2026-2030, 2015 Dec 6.

[12]. C. Wang, Z.Liu, S .C. Chan, "Superpixel-Based Hand Gesture Recognition With Kinect Depth Camera," IEEE Transactions on Multimedia 2015, 17(1) : 29-39.

[13]. Chen WL, WU CH, Lin CH, "Depth-based hand gesture recognition using hand movements and defects," in Next-Generation Electronics (ISNE), 2015 International Symposium, Taiwan Taipei, New York : IEEE 2015, pp. 1-4, 2015 May 4.

[14]. Wong WS, Hsu SC, Huang CL, "Virtual touchpad : Hand gesture recognition for smartphone with depth camera," in Consumer Electronics- Taiwan (ICCE-TW), 2015 IEEE International Conference, Taiwan Taipei, New York : IEEE 2015, pp. 214215, 2015 Jun 6.

[15]. Ishiyama H, Kurabayashi S, "Monochrome glove: A robust real-time hand gesture recognition method by using fabric glove with design of structured markers," in Virtual Reality (VR),
Greenville SC, New York : IEEE 2016, pp.187-188, 2016 Mar 19.

[16]. Davi Hirafuji Neiva, Cleber Zanchenttin, "Gesture recognition : A review focusing on sign language in a mobile context," in Expert system with Applications, 103, 159-183, 2018.

[17]. Suharjito, Ricky Anderson, Fanny Wiryana, Meita Chandra, ariesta, Gede Putra Kusuma, "Sign Language Recognition Application Systems for Deaf-Mute People : A Review Based on InputProcess-Output," in 2nd International Conference on Computer science and Computational Intelligence 2017, ICCSCI 2017, Bail, Indonesia, pp.3-14, October 2017.

[18]. Ming Jin Cheok, Zaid omar, Moha,ed Hisham Jaward, " A review of hand gesture and sign language recognition techniques," 2017.

[19]. Tejashri J, Joshi, shiva Kumar, N. Z. Tarapore, Vivek Mohile, "Static Hand Gesture Recognition using an Android Device," in International Journal Computer Applications (0975 - 8887), Vol 12, No.21, 2015.

[20]. Xinyun Jiang, Wasim Ahmad, "Hand Gesture Detection Based Real-Time American Sign Language Letters recognition using Support Vector Machine," in 2019 IEEE Intl Conf on Dependable Autonomic and secure Computing, Intl Conf on Pervasive intelligence and Computing, Intl conf on Cloud and Big Data Computing, Intl Conf on Cyber Science and TechnologynCongress(DASC/PiCom/CBDCom/Cy berSciTech).

[21]. Ying Xie, Kshitij Bhantupali, "American Sign Language Recognition using Deep Learning and computer Vision.” In 2018 IEEE International Conference on Big Data (Big Data).

Cite this Article : Krutika S. Kale, Prof. Milind B. Waghmare, "Review on Hands Gestures Using American Sign Languages", International Journal of Scientific Research in Computer Science, Engineering and Information Technology (IJSRCSEIT), ISSN : 2456-3307, Volume 7 Issue 3, pp. 228-232, May-June 2021. Available at doi : https://doi.org/10.32628/CSEIT217361 Journal URL : https://ijsrcseit.com/CSEIT217361 